\title{
Politique
}

\section{Le NPD de 1984 à 1988 : la recherche d'un nouvel élan}

\section{André Lamoureux}

Numéro 14, automne 1988

Sport et politique et Le NPD

URI : https://id.erudit.org/iderudit/040602ar

DOI : https://doi.org/10.7202/040602ar

Aller au sommaire du numéro

Éditeur(s)

Société québécoise de science politique

ISSN

0711-608X (imprimé)

1918-6584 (numérique)

Découvrir la revue

Citer cet article

Lamoureux, A. (1988). Le NPD de 1984 à 1988 : la recherche d'un nouvel élan. Politique, (14), 83-118. https://doi.org/10.7202/040602ar d'utilisation que vous pouvez consulter en ligne.

https://apropos.erudit.org/fr/usagers/politique-dutilisation/ 


\title{
LE NPD DE 1984 À 1988: LA RECHERCHE D'UN NOUVEL ÉLAN*
}

\author{
André Lamoureux \\ Cegep André-Laurendeau
}

Le 21 novembre 1988, les Canadiens sont allés aux urnes à l'occasion d'élections fédérales tenues un peu plus de quatre ans après celles du 4 septembre 1984.

La présente étude, consacrée au Nouveau Parti démocratique, a été complétée au début de l'automne 1988, au moment même où ces élections ont été annoncées. Par conséquent, elle ne traite pas des résultats de ces élections qui ont eu pour effet de modifier le cours de la vie politique et l'évolution du NPD au Canada et au Québec. Ce n'est pas non plus l'objectif qui lui a été assigné. Cette recherche est consacrée à l'analyse de la performance du NPD dans la période qui a précédé ces élections, soit au cours des quatre années qui se sont écoulées entre les élections fédérales de 1984 et celles de 1988. L'analyse de cette évolution fournit un éclairage utile sur l'évolution récente de ce parti et sur l'arrièreplan qui a conduit aux résultats de l'élection du 21 novembre 1988.

*NDLR: L'article qui suit traite de l'évolution du NPD au Canada et au Québec de 1984 à 1988. Produit au début de l'automne 1988, il n'intègre pas l'analyse des résultars des élections fédérales du 21 novembre dernier. À la demande de la revue Politique, l'auteur s'est engagé à fournir un complément d'analyse couvrant ces développements récents qui sera publié dans le prochain numéro de la revue.

Politique, 14 (Automne 1988). 
Il est nécessaire de rappeler qu'au lendemain des élections de 1984, à l'approche de ses 25 ans d'existence, le NPD restait marqué par un bilan partagé. D'un côté, après sa création en 1961, le NPD a réussi à devenir un parti solidement implanté au Canada anglais, particulièrement en Ontario et dans trois provinces de l'Ouest: le Manitoba, la Saskatchewan et la ColombieBritannique. Il a trouvé là une base électorale significative, un membership et des appuis solides. Il y a réalisé aussi de bonnes performances électorales. Mais au plan fédéral, le NPD n'a jamais été en mesure de dépasser $20 \%$ des voix et la trentaine de députés. Dans les provinces maritimes, il est toujours resté à la frange de la vie politique et électorale. Au Québec, il n'a jamais pu s'imposer.

Par contre, de 1984 à 1987, le Nouveau Parti démocratique a connu un renforcement significatif de sa cote de popularité dans les sondages et l'opinion publique, au plan canadien comme au plan québécois. Il en est de même pour le chef de ce parti, Ed Broadbent. À vrai dire, après sa fondation, le NPD n'a jamais bénéficié de circonstances semblables à celles qui ont suivi l'élection du 4 septembre 1984. Dans ces conditions, le NPD a cherché à se placer en position de force sur la scène politique canadienne. Il a tout particulièrement tenté de sortir de l'impasse dans laquelle il a été enfermé au Québec depuis sa fondation.

Ce déploiement du NPD de 1984 à 1988 s'est effectué selon de nouvelles données de la situation politique. Après la victoire conservatrice aux élections fédérales du 4 septembre 1984, d'une province à l'autre, les alternances de gouvernements et les revirements de situations ont été nombreux. Plusieurs élections se sont succédées: à Terre-Neuve, en Ontario (en 1985 et en 1987), au Québec, au Manitoba (en 1986 et en 1988), en Alberta, en Saskatchewan, en Colombie-Britannique, au Nouveau-Brunswick, au Yukon et en Nouvelle-Écosse. Celles-ci sont venues modifier les données de la situation politique à la veille d'une nouvelle élection fédérale. 
Au cours de ces quatre années qui ont séparé les élections fédérales du 4 septembre 1984 de celles du 21 novembre 1988, quelle a été la performance du NPD? Quelle trajectoire a-t-il suivie? Y a-t-il eu un réel renforcement de ses assises électorales? Quels enseignements généraux pouvons-nous retenir de cette évolution? La présente étude cherche notamment à vérifier de manière concrète l'apparente popularité du NPD au plan canadien et québécois après 1984. Les pas franchis sont analysés et replacés dans leur contexte politique. Notre recherche analyse aussi les principaux obstacles et enjeux qu'a rencontrés le NPD dans certe progression, particulièrement en rapport avec le Québec.

L'étude rappelle d'abord la performance du NPD au cours de l'élection fédérale de 1984. Elle s'attarde ensuite à vérifier si l'apparente croissance de la popularité du NPD, indiquée après 1984, a reposé sur une tendance véritable. Sous un premier volet, cette analyse est menée en relation avec la scène politique fédérale. Par la suite, notre attention se tourne vers les multiples élections provinciales qui se sont succédées au Canada anglais, afin d'y sonder les performances du NPD. Dans une quatrième étape, l'évolution de la position du NPD sur la question nationale est analysée de manière détaillée, dans la mesure où elle exerce une influence importante sur le développement du NPD au Québec. L'évolution du NPD sur le terrain proprement québécois est l'objet de la dernière partie du texte.

Cette analyse des principales performances du NPD au cours de ces quatre dernières années nous permet de dégager quelques enseignements de premier plan. Ceux-ci fournissent une base essentielle pour une meilleure compréhension de la performance du NPD à l'occasion des élections fédérales du 21 novembre 1988. 


\section{Le rendez-vous de 1984}

Pour une première fois, les élections fédérales du 4 septembre 1984 ont fourni au NPD une ouverture comme il ne s'en était jamais présentée depuis sa fondation en 1961.

En 1984, les libéraux ont vu leurs appuis électoraux s'affaisser, eux qui ont plus ou moins dominé la scène politique fédérale depuis le début des années 1960. Les ressacs de la récession et de la politique monétaire pratiquée par les autorités fédérales, le chômage élevé, la piètre performance de l'économie canadienne, le coup de force constitutionnel de 1981-82 et l'attitude arrogante du gouvernement Trudeau, tout comme la faiblesse de leadership du Parti Libéral nouvellement dirigé par John Turner ${ }^{1}$, tous ces éléments ont certainement contribué à un tel revirement de la situation.

À l'approche de l'échéance électorale, le NPD a donc cru pouvoir capitaliser sur ce nouveau contexte créé par la débâcle libérale. Au point de départ, le parti comptait sur l'appui, plus ou moins manifeste, du Congrès du Travail du Canada. Le NPD a axé sa campagne sur le plein emploi, sur la nécessité de modifier le mode de taxation, sur la sauvegarde des programmes sociaux, comme sur la promotion des droits des femmes. Au Québec, dirigé par Jean-Paul Harney depuis mars 1984, le NPD comptait recueillir $20 \%$ des voix et faire élire de trois à quatre candidats. Mais les conservateurs ont balayé les élections.

Rappelons les résultats de ces élections pour le NPD. En ce qui concerne le suffrage exprimé, le NPD a simplement réussi

1. Au cours de la campagne électorale de 1984, la faiblesse de John Turner comme chef de parti commençait déjà à se manifester. Les bévues ont été nombreuses, dont les tapes au derrière de Iona Campagnolo. Le 4 août 1984, Bill Lee, le directeur de la campagne libérale, démissionnait, confirmant que la machine électorale de John Turner était divisée et connaissait des ratés. Aussi, le 24 août 1984, un sondage Crop-La Presse révélait quà peine $26 \%$ des personnes interrogées pensaient que John Turner ferait le meilleur premier ministre du Canada (alors que son parri recueillait l'appui de $32 \%$ des répondants). 
à maintenir, ou presque, sa performance électorale de 1980. Le 4 septembre 1984 , le NPD récoltait $18,80 \%$ des voix contre $19,77 \%$ en $1980 .^{2}$ Il ne faisait élire que 30 députés, contre 32 en 1980. Dans l'Ouest, le NPD a connu des reculs. En ColombieBritannique, le parti est passé de 12 députés en 1980 à 8 en 1984. En Alberta, l’organisation néo-démocrate n'a pas réussi à percer cette forteresse conservatrice, restant sans député comme toujours depuis 1961. En Saskatchewan, la députation du NPD a aussi baissé, passant de 7 députés en 1980 à 5 en 1984. Au Manitoba, elle a diminué de trois sièges (de 7 à 4). Par contre, en Ontario, le NPD a connu un renforcement de ses positions, particulièrement dans les zones industrielles du Nord de la province où l'implication active des syndicats a joué fortement. En bout de ligne, le NPD s'est assuré de 13 sièges. C'est plus que le double par rapport à l'élection de 1980 , où le parti n'avait recueilli que 5 sièges.

Dans les provinces maritimes, l'héritage du NPD est resté inchangé. Le parti n'a fait élire aucun député. Au Québec, la partie n'a pas été plus facile. Le chef du NPD-Québec, Jean-Paul Harney, était peu connu de la population du Québec. La capacité et l'organisation du parti restaient très faibles; le membership aussi, malgré une légère progression (avec un peu plus de mille membres). Le parti, outre un appui très timide de la FTQ, ne bénéficiait pas non plus du support du mouvement syndical, à la différence du NPD au Canada anglais où les organisations syndicales fournissent une structure et un point d'appui importants. D'autre part, l'organisation néo-démocrate ne pouvait échapper au passé peu reluisant du NPD au Québec sur la question nationale (que nous abordons de manière spécifique et détaillée dans les dernières parties de notre article). Compte tenu de toutes ces circonstances,

2. Selon les données du Directeur général des élections, Elections - Canada. 
le NPD a connu une faible performance au Québec. Il a dû se contenter d'un maigre $8.77 \%$ des voix comparativement à 9,07\% en 1980 .

Tout compte fait, le 4 septembre 1984, c'est Brian Mulroney qui a su canaliser le mouvement d'insatisfaction vis-à-vis des libéraux. Au Québec, bénéficiant de l'appui de René Lévesque et de l'organisation péquiste, Brian Mulroney a utilisé au profit de son parti le fort mécontentement et les frustrations nationales des Québécois engendrées par le coup de force constitutionnel de 1982. Quant à lui, le NPD n'a pas été en mesure de bénéficier de la déroute libérale et n'a pas encore démontré une vigueur nouvelle, si ce n'est en Ontario où les néo-démocrates ont presque supplanté la députation libérale. Au Québec, le vote n'a pas traduit une allégeance plus forte envers ce parti.

\section{Un regain de popularité du NPD?}

Après 1984, toutes les maisons de sondage ont régulièrement sondé les intentions de vote de la population dans l'hypothèse d'une élection fédérale. Des sondages Gallup à ceux de la firme Angus Reid, les pronostics ont été parfois concordants, parfois contradictoires. On sait la valeur relative de ces sondages entre les élections. Ils ne sont pas tenus dans le cadre d'une réelle campagne électorale, avec sa propre dynamique, sa propre mouvance. Malgré tout, les consultations menées par les maisons de sondage, ceux de Gallup en particulier, sont reconnus pour leur niveau de précision élevé.

De 1984 à 1988 , les intentions de vote ont indiqué successivement deux tendances (tableau I). Dans un premier temps, les conservateurs ont vu leurs appuis baisser considérablement. Mais par la suite, notamment à compter de janvier 1988, le Parti conservateur a pu bénéficier d'une remontée de ses appuis dans les sondages. 
Dans une première étape, jusqu'en 1988, la dégringolade du Parti conservateur s'explique dans une large mesure, sans que ce facteur soit exclusif, par le fait que le gouvernement Mulroney se soit enlisé dans de multiples scandales ${ }^{3}$ : celui du thon avarié (qui a mené à la démission du ministre John Fraser), l'affaire Stevens (trouvé coupable 14 fois de conflit d'intérêts), l'affaire Oerlikon (entraînant la démission du ministre André Bissonnette), l'affaire Gravel (accusé de corruption via 50 chefs d'accusation), l'affaire Côté (accusé d'avoir emprunté 250,000 \$auprès d'un homme d'affaire ayant bénéficié de faveurs des conservateurs), etc. Les déboires répétés du gouvernement conservateur ont entraîné un vaste mouvement d'insatisfaction parmi la population. En retour, la cote électorale des libéraux a évolué à la hausse, profitant de cette suite de scandales. De 1985 à 1987, en effet, les intentions de vote en faveur des libéraux se sont fixées entre 35 et $40 \%$ des répondants.

La remontée ultérieure des conservateurs et la baisse des intentions de vote en direction du Parti libéral s'explique surtout, à notre avis, par la faiblesse de John Turner à titre de chef de parti et par la division interne qui a traversé cette organisation. En 1988, cette faiblesse de leadership s'est transformée en une défaillance de premier plan ${ }^{4}$, dont Brian Mulroney a su profiter tout en distribuant de gauche à droite les projets gouvernementaux, les subventions et les promesses pré-électorales.

3. En août 1986, une porte-parole de la maison Gallup, Mme Clara Hatton, cherchant à expliquer la situation, évoquait même un phénomène d'écœurement à l'endroit du gouvernement Mulroney. La Presse, 22 août 1986.

4. Cette crise de leadership au sein du Parti libéral s'est d'abord manifestée en novembre 1986 alors que Marc Lalonde appelait à un changement de chef. À l'automne 1987, un groupe de députés, majoritairement du Québec, contestait le leadership de John Turner devant l'impopularité et la mauvaise performance de leur chef. Précisons que dans son livre publié en septembre 1988 sous le titre "Reign of error", le journaliste et chroniqueur parlementaire Greig Weston, pour sa part, décrivait le chef libéral comme un dirigeant incompétent et indécis. 
Tableau 1

\section{LA POSITION DES TROIS GRANDS PARTIS FÉDÉRAUX DANS LES} SONDAGES GALLUP, DE SEPTEMBRE 1984 À SEPTEMBRE 1988.

$\grave{A}$ chaque occasion, la question posée était la suivante: «Si une élection fédérale avait lieu aujourd'hui, quel serait le parti de votre choix".

Les résultats sont présentés en pourcentage. Ils n'indiquent pas la part des indécis.

\begin{tabular}{|c|c|c|c|}
\hline Sondages & $P C$ & PLC & $N P D$ \\
\hline Sondage pré-électoral 1984 (27-29 août) & 50 & 28 & 19 \\
\hline Élections du 4 sept. 1984 & 50 & 28 & 19 \\
\hline 11-13 octobre 1984 & 60 & 21 & 17 \\
\hline I-3 novembre 1984 & 55 & 23 & 20 \\
\hline 6-8 décembre 1984 & 54 & 24 & 20 \\
\hline $3-5$ janvier 1985 & 53 & 25 & 21 \\
\hline 31 janv. -2 fév. 1985 & 53 & 25 & 21 \\
\hline $21-23$ février 1985 & 56 & 26 & 17 \\
\hline $4-6$ avril 1985 & 54 & 24 & 21 \\
\hline 16- 18 mai 1985 & 45 & 31 & 22 \\
\hline $6-8$ juin 1985 & 44 & 33 & 21 \\
\hline $11-13$ juillet 1985 & 40 & 33 & 26 \\
\hline $9-10$ août 1985 & 46 & 32 & 20 \\
\hline 5-7 septembre 1985 & 48 & 29 & 22 \\
\hline 3-5 octobre 1985 & 43 & 35 & 22 \\
\hline $7-9$ novembre 1985 & 40 & 36 & 24 \\
\hline 5-7 décembre 1985 & 37 & 38 & 24 \\
\hline $2-4$ janvier 1986 & 41 & 36 & 23 \\
\hline 6-8 février 1986 & 36 & 41 & 23 \\
\hline 13-15 mars 1986 & 41 & 34 & 25 \\
\hline $3-5$ avril 1986 & 37 & 40 & 21 \\
\hline 1-3 mai 1986 & 37 & 41 & 21 \\
\hline $5-7$ juin 1986 & 32 & 40 & 27 \\
\hline 3-5 juillet 1986 & 36 & 41 & 21 \\
\hline $7-9$ août 1986 & 31 & 35 & 33 \\
\hline 14-16 août 1986 & 33 & 41 & 24 \\
\hline 11-13 septembre 1986 & 35 & 36 & 28 \\
\hline $2-4$ octobre 1986 & 31 & 38 & 29 \\
\hline 6-8 novembre 1986 & 31 & 39 & 29 \\
\hline 3-6 décembre 1986 & 30 & 45 & 25 \\
\hline 7-10 janvier 1987 & 28 & 41 & 30 \\
\hline 11-14 février 1987 & 22 & 44 & 32 \\
\hline $11-14$ mars 1987 & 24 & 41 & 34 \\
\hline $8-11$ avril 1987 & 24 & 42 & 32 \\
\hline 6-9 mai 1987 & 26 & 42 & 30 \\
\hline $3-6$ juin 1987 & 24 & 39 & 35 \\
\hline $8-11$ juillet 1987 & 23 & 35 & 41 \\
\hline
\end{tabular}


Sondages

5-8 août 1987

$P C$

PLC

$N P D$

2-5 septembre 1987

25

36

37

7-10 octobre 1987

4-7 novembre 1987

2-5 décembre 1987

6-9 janvier 1988

3-6 février 1988

2-5 mars 1988

6-9 avril 1988

4-7 mai 1988

1-4 juin 1988

6-9 juillet 1988

Source: La Presse.

Ce qui reste étonnant, c'est la performance du NPD de 1984 à 1988. Après 1984, le NPD s'est affirmé considérablement dans les intentions de vote de la population canadienne. Même si le mouvement a connu des irrégularités, la tendance à la hausse a été indiquée, du moins jusqu'en 1988. Mais cette affirmation du NPD dans les sondages à l'échelle du pays est en bonne partie imputable à la hausse manifestée au Québec, un fait nouveau qui a influencé le cours des sondages au plan canadien. Au cours de l'année 1987, les intentions de vote en faveur du NPD au Québec se sont situées au-dessus de $30 \%$ et ont même atteint $40 \%$ en juillet et en septembre 1987, selon Gallup.

Par contre, au cours de l'année 1988, les appuis du NPD ont relativement diminué dans les sondages. Le Québec a encore une fois contribué à ce mouvement à la baisse, alors que les appuis du NPD y ont chuté à $27 \%$ en février 1988 et à $26 \%$ en mai. Cette baisse s'est exprimée au fur et à mesure que les conservateurs voyaient leurs appuis remonter. Mais de façon générale, les intentions de vote en faveur du NPD au Canada se sont maintenues audessus de $25 \%$, de l'été 1986 à septembre 1988. Au Québec, il s'agissait d'un phénomène sans précédent. 
Face à cette tendance manifestée après 1984 en faveur du NPD, une interrogation ressurgit. L'apparente popularité du NPD a-t-elle reposé sur une tendance véritable?

On a souvent dit que la popularité croissante du NPD après 1984 n'était qu'illusoire. Le NPD n'aurait été populaire que dans les sondages et entre les élections, mais pas au moment du vote. ${ }^{5}$ Il serait aussi permis de croire que l'apparente vigueur du NPD n'ait été attribuable qu'à des facteurs étrangers à sa propre performance. Elle s'expliquerait uniquement par défaut, comme simple résultante de l'impopularité des conservateurs et de la faiblesse circonstancielle de leurs adversaires, manquant de cohésion et divisés quant au leadership de John Turner. ${ }^{6}$

Sur ces deux facettes de la question, analysons la situation de plus près, d'abord sur la scène fédérale.

Au plan fédéral, le phénomène de la popularité montante du NPD a dépassé le simple niveau des intentions au cours de l'année 1986 et celle de 1987. Les cinq élections partielles tenues pendant cette période en sont une bonne illustration: d'abord dans les comtés de Pembina (Alberta) et de Saint-Maurice (Québec), le 29 septembre 1986; par la suite, le 20 juillet 1987, à l'occasion des trois élections partielles tenues dans les comtés d'Hamilton Mountain (Ontario), celui de Saint-Jean Est (Terre-Neuve) et celui du Yukon. Toutefois, lors de la seule élection partielle de l'année 1988, celle tenue dans le comté de Lac-Saint-Jean le 20 juin, le NPD a connu une faible performance. Ce résultat est venu assombrir les gains marqués par le NPD au Québec en 1987, dans le comté de Saint-Maurice.

5. Voir, entre autres, un éditorial de Michel Roy intirulé «La vigueur du NPD", La Presse, le 30 octobre 1986.

6. C'est notamment l'opinion rendue par Vincent Lemieux à l'automne 1986. Voir le premier article d'une série de trois, écrit par le journaliste Bernard Descoteaux et intitulé «Le Nouveau Parti Démocratique et le Québec: Une percée encore fragile», Le Devoir, le 25 septembre 1986. 
Une brève analyse de ce cheminement du NPD au plan fédéral s'impose.

Le 29 septembre 1986, dans le comté de Pembina, le résultat de l'élection partielle a surpris. Il importe de rappeler qu'en 1984, les conservateurs avaient remporté haut la main les 21 comtés de la province avec des majorités dépassant souvent les 20,000 ou 30,000 voix. Le jour du vote, le candidat du NPD, Ivor Dent, a recueilli $36,41 \%$ des voix $(16,251)$ contre $37,02 \%(16,525)$ pour le candidat conservateur élu, Walter Van de Walle. ${ }^{7}$ Il suffisait de peu pour que le NPD rafle le comté. Par comparaison, en septembre 1984, dans ce même comté, le candidat conservateur Peter Elzinga avait battu son adversaire néo-démocrate par une majorité de 34,067 voix. Il s'agissait donc d'un revirement de situation, manifesté à l'échelle d'un comté, dans une province considérée comme un château fort pour les conservateurs.

Dans le comté de Saint-Maurice, le candidat du NPD a aussi fait bonne figure, surtout si l'on considère l'héritage du NPD au Québec. Tel que prévu, l'élection dans cet ancien fief de Jean Chrétien a été remportée par les libéraux. Mais le candidat du NPD, Claude Rompré, a livré une lutte serrée au candidat conservateur pour l'obtention de la deuxième place. En fin de course, le candidat néo-démocrate a récolté $19,48 \%$ des voix contre $20,2 \%$ pour son adversaire, une différence de 182 voix seulement. Par rapport à 1984, le NPD a presque quintuplé son support électoral dans ce même comté.

$\mathrm{Au}$ cours de ces deux premières élections partielles, les conservateurs ont donc conservé le comté de Pembina (de justesse) et les libéraux le comté de Saint-Maurice. Mais les appuis électoraux du NPD se sont affirmés. En quelque sorte, ces élections partielles annonçaient, par leurs résultats, celles d'Hamilton Mountain, de Saint-Jean Est et du Yukon.

7. Données fournies par Élections - Canada, Ottawa. 
Le 20 juillet 1987, le NPD remportait la victoire dans ces trois autres comtés. Dans la circonscription d'Hamilton Mountain, malgré une nette progression du vote libéral, la candidate Marion Dewar réussissait à conserver le comté au NPD. Les résultats les plus étonnants sont ceux de Saint-Jean et du Yukon, deux comtés remportés par les conservateurs en 1984 avec une très forte majorité. Dans le comté de Saint-Jean, le candidat néo-démocrate, Jack Harris, a recueilli $46,39 \%$ des voix en comparaison avec le maigre $6,56 \%$ récolté par le NPD en $1984 .{ }^{8}$ C'est un contraste frappant. Dans le comté du Yukon, la candidate Audrey McLaughlin amenait aussi le NPD à la victoire, avec 35,64\% des voix, contre $16,10 \%$ pour Sybil Frei en 1984.

Les cinq élections partielles fédérales tenues en 1986 et 1987 confirmaient donc la nouvelle popularité du NPD indiquée par les sondages. De plus la vigueur montante du NPD en 1986 et en 1987 ne semblait pas être due aux seuls déboires des conservateurs et à la seule défaillance des libéraux. D'une part, la popularité personnelle d'Ed Broadbent a connu une croissance très nette au cours de ces années 9 . De plus, les interventions publiques multipliées du NPD pendant la même période ont possiblement contribué à cette hausse de popularité. Signalons les déclarations répétées du NPD révélant les privilèges fiscaux des corporations, dont la publication de la retentissante Enquête fiscale' 86 . Dans cette enquête, le NPD démontrait que certaines des plus grandes et des plus rentables des sociétés du pays ne payaient pas d'impôts, comme la société Argus et la société Power en 1985. Ces révélations, qui ont connu un impact public important, ont même ultérieurement obligé le gouvernement Mulroney à présenter un projet de réforme fiscale. Notons aussi les déclarations du NPD dirigées contre le libre échange ou celles consacrées à la défense des programmes

8. Selon les données fournies par Élections - Canada.

9. Popularité confirmée par tous les sondages tenus pendant cette période. 
sociaux, comme l'indexation des pensions de vieillesse et des allocations familiales mise en cause par le gouvernement Mulroney. Le discours tenu par le NPD a vraisemblablement rejoint les frustrations et les attentes des groupes les plus démunis, d'une foule de ménages, de travailleurs, de syndicalistes, et même de certaines couches des classes moyennes. Fait indicatif, en septembre 1987, un sondage mené par la maison Angus Reid démontrait que, pour une première fois après 1985 , les opposants au libre échange prenaient un pas d'avance devant ses partisans parmi la population canadienne ${ }^{10}$.

Au cours de l'année 1986 et celle de 1987, la cote électorale du NPD a donc évolué à la hausse. Toutefois, l'élection partielle tenue dans le comté de Lac-Saint-Jean en juin 1988 n'a pas permis au NPD de confirmer cette vigueur nouvelle. Lucien Bouchard, candidat-vedette des conservateurs, a remporté cette élection avec $55,16 \%$ des voix exprimées, contre $34,8 \%$ pour le candidat libéral Pierre Gimaïel et 9,62 \% pour Jean Paradis, candidat du NPD $^{11}$. Cette victoire a eu un caractère nettement particulier. Lucien Bouchard avait appelé à voter OUI au référendum québécois de 1980 et se présentait dans un comté fortement nationaliste. Aussi, il bénéficiait de l'appui de Robert Bourassa, des dirigeants du Parti québécois, et de plusieurs personnalités, dont Corinne Côté-Lévesque. Lucien Bouchard comptait aussi sur son poste ministériel et sur des promesses électorales de plus de 4 millions de dollars en subventions aux entreprises et aux projets municipaux dans le comté. Bref, il n'y avait rien de très surprenant dans la victoire de Lucien Bouchard. Quant au NPD, sa performance s'est avérée plutôt faible. Jean Paradis a amélioré légèrement la performance du NPD en comparaison avec l'élection de septembre 1984 où le parti n'avait recueilli que $5,2 \%$ des voix dans ce

10. Rapporté par le journal Le Devoir, le 9 septembre 1987.

11. Selon Élections - Canada. 
même comté. Mais le 20 juin 1988, le score du NPD est demeuré sous la barre des $10 \%$. Ce résultat, en dépit des conditions toutes particulières de la victoire de Lucien Bouchard, démontrait que rien n'était gagné d'avance au Québec pour le NPD à l'approche de l'élection fédérale de 1988. Ceci reste d'autant plus vrai que ce résultat survenait dans un contexte où les appuis populaires du NPD dans les sondages amorçaient un mouvement à la baisse en comparaison avec ce que le parti avait connu en 1987. La performance du candidat néo-démocrate démontrait surtout que la popularité personnelle d'Ed Broadbent ne pouvait être garante, à elle seule, de la construction du NPD au Québec.

D'autre part, pour bien saisir la progression du NPD au plan canadien de 1984 à 1988, il importe de mesurer si ce parti a connu une croissance de ses appuis dans les diverses élections provinciales tenues au Canada anglais après l'élection du 4 septembre 1984.

Au Canada anglais: le test des élections provinciales

Après 1984, plusieurs élections provinciales se sont succédées au Canada anglais, dans l'Ouest canadien comme dans les provinces maritimes. L'analyse des résultats de ces élections provinciales permet de voir si la popularité montante du NPD au plan fédéral a connu sa traduction dans ces mêmes provinces.

En fait, la trajectoire suivie par le NPD dans les provinces $\mathrm{du}$ Canada anglais indique un mouvement en deux temps. Dans une première étape, au cours de l'année 1985 et celle de 1986 , le NPD semble avoir bénéficié de sa vague de popularité montante au plan canadien. À l'occasion d'élections provinciales tenues à Terre-Neuve, en Ontario, au Manitoba, en Alberta, en Saskatchewan et en Colombie-Britannique, le NPD a effectivement marqué des points. Il a aussi renforcé ses positions au Yukon en avril 1985. Par contre, au cours de la période qui a suivi, les performances 
du NPD en Ontario, au Nouveau-Brunswick au Manitoba et en Nouvelle-Écosse ont été moins reluisantes. L'examen de ce mouvement contradictoire permet d'évaluer les points forts et les points faibles de l'évolution du NPD après 1984.

Sous un premier volet, examinons et analysons les gains effectués par le NPD dans certaines provinces du Canada anglais au cours des deux années 1985 et 1986.

Le premier rendez-vous fut celui de Terre-Neuve, en avril 1985. Au cours des élections générales tenues le 2 avril, le NPD a consolidé ses appuis chez la population terre-neuvienne affectée par le chômage et de plus en plus mécontente des politiques économiques du gouvernement Peckford ${ }^{12}$. Il est parvenu à presque quadrupler les voix obtenues en 1982. Ses appuis sont passés à $14,3 \%$ du vote populaire, contre $4 \%$ en 1982 . Le parti a fait élire deux députés, contre un seul en 1982. ${ }^{13}$

Deux semaines plus tard, le NPD gagnait les élections législatives au Yukon. ${ }^{14}$

En Ontario, le 2 mai 1985, après avoir dominé la province depuis 1943, la machine conservatrice a cédé le pas devant la poussée du vote libéral et néo-démocrate. Les libéraux de David Peterson sortaient grands gagnants du vote au suffrage populaire, bien qu'ils aient obtenu 4 députés en moins que les conservateurs (48 contre 52). Néanmoins, le NPD profitait aussi légèrement de cette volonté de changement en renforçant ses positions dans quelques comtés industriels et urbains de la province (Témiskaming,

12. "Terre-Neuve: le gouvernement Peckford réélu avec une majorité réduite», Le Devoir, 3 avril 1985

13. À Terre-Neuve, comme dans les autres provinces maritimes, le NPD ne compte qu'une petite organisation. En 1987, il ne comptait que 1,200 membres, soit encore moins que le NPD-Québec.

Source: «Les Néo-démocrates au Canada» dans une brochure intitulée: On prend l'avenir en main, publiée par le NPD à l'occasion du congrès fédéral de mars 1987, p. 16.

14. À ce propos, voir Morton Desmond, The Neu Democrats 1961-1986. The Politics of Change, Toronto, Coop Clark Pitman Ltd., 1986, p. 223. 
Oshawa, Ottawa centre, etc.) Comparativement à 1981, le vote en faveur du NPD évoluait de $21,1 \%$ à $23,8 \%$. La députation néo-démocrate grimpait à 25 sièges, quatre de plus qu'en 1981 . Par la suite, le NPD décidait de se liguer aux libéraux, minoritaires à l'Assemblée législative, pour leur permettre de former le gouvernement. Ils s'engageaient à soutenir le gouvernement libéral de David Peterson pour une période de deux ans. En retour, les libéraux acceptaient de réaliser un programme de réformes proposé par le NPD.

\begin{tabular}{|c|c|c|c|c|c|c|}
\hline \multicolumn{7}{|c|}{$\begin{array}{l}\text { Les élections en Ontario } \\
\text { (1981-1985 et 1987) }\end{array}$} \\
\hline \multicolumn{7}{|c|}{$\begin{array}{l}\text { Résultats des trois grands partis: } \\
\text { pourcentage des voix recueillies et nombre de sièges obtenus. }\end{array}$} \\
\hline & \multicolumn{2}{|c|}{19 mars 1981} & \multicolumn{2}{|c|}{2 mai 1985} & \multicolumn{2}{|c|}{10 septembre 1987} \\
\hline & \multicolumn{2}{|c|}{$\%$ voix/sièges } & \multicolumn{2}{|c|}{ \% voix/sièges } & \multicolumn{2}{|c|}{ \% voix/sièges } \\
\hline NPD & $21,1 \% *$ & 21 & $23,8 \%$ & 25 & $25,7 \%$ & 19 \\
\hline Parti conservateur & $44,4 \%$ & 70 & $37,0 \%$ & 52 & $24,7 \%$ & 16 \\
\hline Parti libéral & $33,7 \%$ & 34 & $37,9 \%$ & 48 & $47,3 \%$ & 95 \\
\hline \multicolumn{2}{|l|}{ Total des sièges: } & 125 & & 125 & & 130 \\
\hline \multicolumn{7}{|c|}{$\begin{array}{l}\text { Sources: Bureau du directeur général des élections, Ontario. } \\
\text { * les pourcentages sont calculés à partir du total des voix exprimées. }\end{array}$} \\
\hline
\end{tabular}

Par la suite, en 1986, les quatre provinces de l'Ouest sont passées aux urnes.

Le Manitoba a donné le coup d'envoi. Le 18 mars, malgré une légère baisse de ses appuis au vote populaire et bien que talonné par le Parti conservateur, le NPD, dirigé par Howard Pawley, a réussi à préserver sa victoire de novembre 1981. Même s’il a été secoué par la crise linguistique manitobaine de 1983 , le gouvernement Pawley est parvenu à renouveler son mandat en 
s'appuyant sur un programme de réformes et un vaste projet de développement hydro-électrique (Morton, 1986, 225).

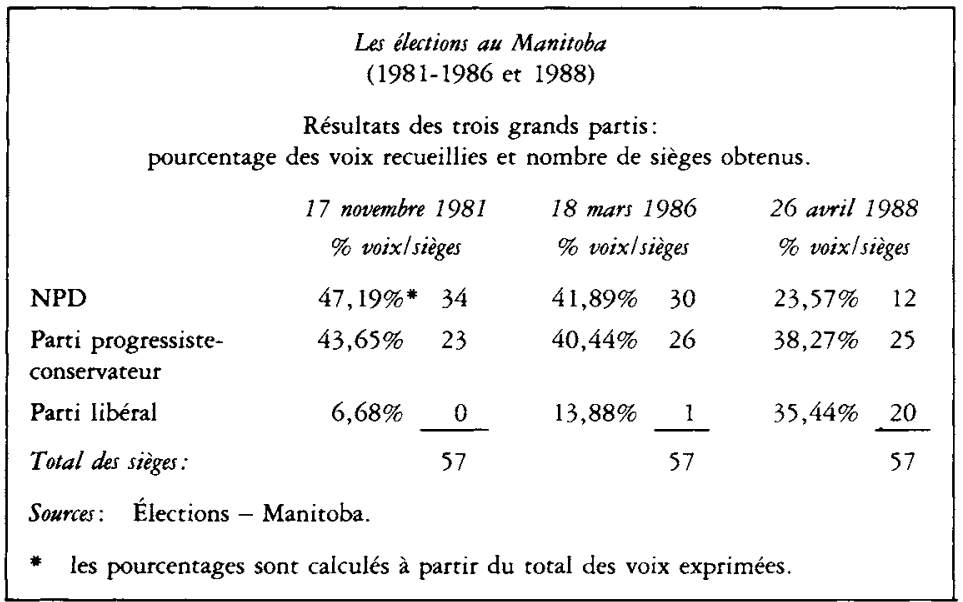

Deux mois plus tard, ce fut au tour de l'Alberta. Confirmant les gains effectués à l'occasion de l'élection partielle fédérale de Pembina, le NPD y a effectué une percée significative en recueillant $29 \%$ des voix. Il a doublé son vote populaire par rapport à l'élection précédente et sa députation est passée de deux à seize. Cette performance est un fait sans précédent de l'histoire du NPD dans cette province. Plusieurs facteurs semblent avoir contribué à cette percée du NPD : la détérioration de la situation économique dans cette province depuis le début des années 1980, la fin des méga-projets dans l'industrie pétrolière, les fermetures d'entreprises, les faillites bancaires, ainsi que les lois albertaines de 1983, interdisant le droit de grève aux employés du secteur public et le remplaçant par l'arbitrage obligatoire. Ces événements ont suscité un mouvement d'insatisfaction face aux gouvernements Lougheed et Getty. Ce changement a largement profité au NPD, particu- 
lièrement en zones urbaines, où le NPD a obtenu la majorité de ses sièges, notamment à Edmonton (Morton, 1986, 226).

Le 20 octobre 1986, en Saskatchewan, le NPD a aussi fait bonne figure. Les conservateurs de Grant Devine ont conservé le pouvoir de justesse. Ils ont réussi, en effet, à maintenir une majorité de sièges à la législature provinciale, comptant sur 38 députés contre 25 pour le NPD. Mais ce n'est là qu'un résultat déformant. Celui-ci est tout simplement imputable au caractère non-proportionnel du système électoral. Le NPD est sorti bel et bien vainqueur de l'élection sur le plan du suffrage populaire: 45,2\% contre $44,6 \%$ pour les conservateurs. Tout compte fait, dans cette province, la force du NPD s'est affirmée en comparaison avec l'élection d'avril 1982. À ce moment-là, des sections entières du mouvement syndical, particulièrement les travailleurs du secteur public et parapublic, avaient boudé le gouvernement néo-démocrate d'Allen Blakeney pour protester contre les lois spéciales et les conditions de travail qui leur avaient été imposées par voie législative (Morton, 1986, 209). Le 26 avril 1982, le parti avait dû se

\section{Les élections en Saskatchewan \\ (1982 et 1986)}

Résultats des trois grands partis: pourcentage des voix recueillies et nombre de sièges obtenus.

NPD

26 avril 1982

\% voix/sièges

20 octobre 1986

\% voix/sièges

Parti conservateur

$37,64 \% * 9$

$45,02 \% \quad 25$

Parti libéral

$54,07 \% 55$

$44,61 \% \quad 38$

Total des sièges:

$4,51 \% \frac{0}{64}$

$9,99 \% \frac{1}{64}$

Sources: Électoral Office, Saskatchewan.

* les pourcentages sont calculés à partir du total des voix exprimées. 
contenter de $37 \%$ des voix et de neuf sièges. Le résultat de 1986 , quant à lui, indiquait que les plaies de 1982 s'étaient peu à peu refermées. L'élection s'est tenue dans un contexte différent, marqué par les difficultés rencontrées par les fermiers de la province et les pourparlers sur le libre échange. Le NPD, toujours dirigé par Allen Blakeney, a mené une forte campagne contre le gouvernement Devine et s'est progressivement assuré d'une nouvelle popularité.

Le 22 octobre 1986, aux élections de Colombie-Britannique, les créditistes ont été reportés au pouvoir à leur tour. Pour leur part, les libéraux ont connu une légère progression. Mais le NPD a maintenu ses positions. Malgré une légère baisse dans le pourcentage des voix obtenues, le NPD a protégé ses acquis. Il conserve dans cette province une force relativement intacte. Le NPD s'appuie encore fortement sur les organisations syndicales qui ont été la cible privilégiée du gouvernement créditiste après 1983.

\section{Les élections en Colombie-Britannique \\ (1983 et 1986)}

Résultats des trois grands partis: pourcentage des voix recueillies et nombre de sièges obtenus.

\begin{tabular}{lrrrr} 
& 5 mai 1983 & 22 actobre & 1986 \\
& $\%$ voix/sièges & \multicolumn{2}{c}{$\%$ voix/sièges } \\
NPD & $44,94 \%$ * & 22 & $42,06 \%$ & 22 \\
Parti créditiste & $49,76 \%$ & 35 & $49,32 \%$ & 47 \\
Parti libéral & $2,89 \%$ & 0 & $6,74 \%$ & 0 \\
\cline { 2 - 4 } Total des sièges: & 57 & & 69 \\
Sources: Élections - British Columbia & & & \\
$*$ & les pourcentages sont calculés à partir du total des voix exprimées.
\end{tabular}

En définitive, dans plusieurs provinces du Canada anglais, des signes de renforcement se sont manifestés pour le NPD en 1985 et 1986. Les libéraux ont aussi connu une croissance de 
leurs appuis électoraux pendant cette même période. Mais la performance du NPD a été notable. Il serait exagéré de comparer ce regain de popularité à la montée qu'a connue le NPD au début des années 1970, alors qu'il a remporté successivement trois élections provinciales, au Manitoba, en Saskatchewan et en ColombieBritannique. Par contre, un mouvement de renforcement a été indiqué, du moins momentanément.

Toutefois, les deux années suivantes se sont présentées sous un jour différent. Tour à tour, en Ontario, au Nouveau-Brunswick, au Manitoba et en Nouvelle-Écosse, les néo-démocrates ont connu de sérieuses difficultés.

Le 10 septembre 1987, David Peterson a assuré aux libéraux ontariens une victoire prépondérante avec $47,3 \%$ des suffrages exprimés et 95 sièges sur un total de 130. Avec cette victoire, les libéraux ont asséné un dur coup aux conservateurs de Jerry Grossman. Ces derniers se sont retrouvés en troisième place pour une première fois depuis 1919 , avec $24,7 \%$ des voix et 16 sièges. De son côté, malgré la vague libérale, le NPD a réussi à améliorer légèrement ses appuis au suffrage populaire. De 1985 à 1987, le vote néo-démocrate a progressé de $23,8 \%$ à $25,7 \%$. Le parti s'est classé au deuxième rang dans 44 des 130 circonscriptions que compte la province. Mais la députation du NPD est passée de 25 sièges en 1985 à 19 en 1987 . Il semble bien qu'après deux ans d'appui au gouvernement de David Peterson, la stratégie du NPD ait davantage profité à leurs adversaires, le chef libéral en ayant recueilli le bénéfice. ${ }^{15}$ En effet, les quelques réformes exigées par le NPD, reprises par le gouvernement Peterson en vertu de l'entente de 1985, ont rapporté des dividendes aux libéraux, et

15. Sur ce plan, certains parallèles peuvent sûrement être établis avec les événements survenus sur la scène fédérale au début des années 1970. De 1972 à 1974, en effet, la stratégie d'appui au gouvernement Trudeau pratiquée par le NPD avait surtout profité aux libéraux fédéraux. Cette stratégie du NPD, dirigé par David Lewis, avait entraîné la débâcle du parti aux élections de 1974 . 
non au NPD. D'autre part, le chef du NPD ontarien, Bob Rae, a concédé la victoire aux libéraux dès le départ de la campagne électorale. Cette stratégie a immanquablement placé le NPD en situation défensive, au lieu de capter l'attention de l'électorat sur l'action propre ou le programme de gouvernement du NPD. Enfin, les libéraux, contrairement au NPD, ont pu compter sur de forts appuis parmi les Franco-ontariens (suite aux législations linguistiques de 1986).

Au Nouveau-Brunswick, après l'Ontario, les libéraux provinciaux de Franck McKenna ont balayé les élections en s'emparant de chacun des 58 comtés de la province. Le 13 octobre 1987, les libéraux ont mis fin à 17 ans de régime conservateur. Le Parti libéral a recueilli $60 \%$ des voix exprimées. ${ }^{16}$ Il a compté, entre autres, sur l'appui de l'Acadie francophone. Dans les comtés francophones du Nord-Est du Nouveau-Brunswick, le Parti libéral a en effet bénéficié de très fortes majorités. Pour sa part, le vote conservateur s'est affaissé à $28.41 \%$ tandis que le NPD a vu ses appuis populaires stagner à $10,48 \%$, quasiment le même vote qu'aux élections du 12 octobre 1982.

Parti à faible rayonnement, anglophone, peu implanté parmi les Acadiens, trouvant ses assises électorales dans les zones urbaines du Sud de la province, le NPD n'a pas été en mesure d'élargir ses appuis. Le parti a été cantonné à de piètres résultats, même s'il a présenté, pour la première fois de son histoire, des candidats dans tous les comtés. George Little, le chef du parti, a été défait dans son propre comté (King-Ouest). Ainsi, l'éclatante victoire libérale a toralement éclipsé les efforts du NPD dans cette province.

Quant au Manitoba, les acquis préservés par le NPD en 1986 se sont effondrés à l'occasion des élections du 26 avril 1988.

16. Rapport du directeur général des élections du Nouveau-Brunswick, février 1988 , p. 32. Les autres données de l'élection du 13 octobre 1987 sont aussi tirées de ce rapport. Le taux de participation à l'élection a été particulièrement élevé, soit $81,95 \%$. 
Le gouvernement Pawley a dû céder le pouvoir. En campagne électorale, les conservateurs de Gary Filmon ont pris les devants pour finalement remporter les élections avec $38,27 \%$ des voix et 25 députés, et former un gouvernement minoritaire. Les libéraux de Sharon Carstairs ont suivi de près en s'assurant 35,44\% des voix et 20 sièges. Pour sa part, dirigé par un nouveau chef désigné quelques semaines à peine avant les élections, affaibli par des mesures impopulaires promulguées par le gouvernement Pawley, le NPD est tombé à son plus faible résultat électoral depuis 20 ans, ne recueillant que $23,57 \%$ des voix et 12 sièges.

Enfin, en Nouvelle-Écosse, le NPD s'est avéré incapable de consolider ses positions à l'occasion de l'élection du 6 septembre 1988, tenue à la veille du scrutin fédéral. Comme au Manitoba, les conservateurs ont été reportés au pouvoir de justesse, avec une majorité de deux sièges seulement (28 sur 52). Même si le gouvernement Buchanan avait été secoué par les scandales ${ }^{17}$, le NPD n'a pas su profiter de la situation, recueillant le même pourcentage des voix exprimées qu'à l'élection de 1984 (16\%) et seulement deux sièges, contre trois à l'élection précédente. Comme au Manitoba, ce sont les libéraux qui ont effectué la plus importante percée, avec $39,5 \%$ des voix et 21 sièges (contre $30 \%$ des suffrages et 6 sièges en 1984).

Ainsi, après avoir effectué quelques gains en 1985 et en 1986, le NPD a ultérieurement connu des difficultés dans plusieurs provinces du Canada anglais. Dans les Maritimes, il n'a guère fait mieux que dans le passé.

De manière générale, on peut retenir que la hausse de popularité du NPD au plan fédéral, quelque soit son ampleur ou ses fluctuations, n'est pas garante d'une meilleure performance

17. Voir le résumé de ces scandales fait par le journal La Presse du 7 septembre 1988 , dans un article intitulé «Buchanan est réélu, mais il perd des plumes». 
du NPD dans les provinces, où la vie politique évolue selon les conditions propres à chacune d'entre elles. Les performances du NPD dans les sondages et les cinq élections partielles fédérales tenues en 1986 et 1987 n'ont pas empêché les reculs subis en Ontario, au Nouveau-Brunswick, au Manitoba et en NouvelleÉcosse. Là, les libéraux ont nettement pris les devants.

Ces constatations recouvrent une valeur d'enseignement tout aussi pertinente pour le Québec, d'autant plus que la question nationale y complique la situation.

\section{La question nationale et l'enjeu constitutionnel}

À compter de 1984, la relance de l'organisation néo-démocrate au Québec s'est avérée le principal déf que le NPD a cherché à relever. Un défi de taille. De 1961 à 1984, en effet, le Québec a sans cesse été le talon d'Achille du parti. L'impasse dans laquelle le NPD a été enfermé a toujours constitué pour lui un handicap majeur, l'empêchant d'envisager toute possibilité de former le gouvernement à Ottawa. Après 1984, le sort du NPD restait donc plus que jamais conditionné à sa progression et à ses performances en territoire québécois. Or, la tentative de relance du NPD au Québec après 1984 ne peut être appréciée qu'en rapport avec la question nationale, sur laquelle le parti a achoppé constamment après sa fondation. Au cours des années 1960 et 1970 , l'orientation du NPD sur cet enjeu lui a enlevé toute possibilité d'une construction réelle au Québec, dans un contexte d'affirmation des aspirations nationales du peuple québécois et alors que le Parti québécois lui coupait l'herbe sous le pied. L'orientation fédéraliste et centralisatrice de la direction fédérale du NPD, sa position en faveur du NON au référendum de 1980 et son appui au coup de force constitutionnel de 1981-82, ont tour à tour miné les efforts de construction du parti au Québec. Pendant cette période, la question nationale s'est vite avérée l'une des raisons principales 
de ses échecs au Québec ${ }^{18}$. En 1985, dans ses efforts de relance, le NPD misait sur le mouvement de désaffection manifesté à l'égard du Parti québécois. Escomptant élargir le nombre de ses supporteurs et surtout gagner le vote nationaliste, Jean-Paul Harney et son équipe ne pouvaient esquiver la question nationale. L'héritage du parti en cette matière n'était guère susceptible de séduire la clientèle nationaliste. Devant ce dilemme, le NPD-Québec a d'abord cherché à se démarquer du passé et de l'orientation connue de l'aile fédérale du parti, de manière à dénouer l'impasse et à trouver de nouveaux appuis chez le peuple québécois. Ce développement a provoqué de nouveaux démêlés avec l'aile fédérale du parti et de nouvelles divisions. L'analyse de ce cheminement permet de mieux apprécier les efforts du NPD au Québec et de voir jusqu'à quel point le NPD a manifesté une ouverture envers les aspirations nationales des Québécois à la veille des élections fédérales de 1988.

Les positions mises de l'avant par le NPD-Québec se rapportent principalement à la question de l'assemblée constituante, au dossier de la langue et à l'Accord du Lac Meech. Sur ces trois volets, le NPD-Québec a développé des positions nouvelles qui n'ont d'ailleurs pas manqué de susciter des réactions à Ottawa.

À ses congrès de mars et de septembre 1985, l'organisation québécoise s'est d'abord prononcée en faveur du droit à l'autodétermination du peuple québécois et de la convocation d'une assemblée constituante. Selon l'orientation établie par le NPDQuébec, cette assemblée, désignée par élection, serait chargée d'établir une constitution proprement québécoise. Ce projet de constitution serait ultérieurement ratifié par référendum et demeurerait souverain sur toute autre loi. La position élaborée par

18. À ce propos, voir l'étude de cette question que l'auteur a effectuée dans le livre Le NPD et le Québec, 1958-1985, Éditions du Parc, 1985, 230 p. 
le NPD-Québec à son congrès de septembre 1985 déclare illégitime la Loi constitutionnelle de 1982, adoptée sans que le peuple québécois n'ait été consulté. ${ }^{19}$

Cette position en faveur d'une assemblée constituante québécoise a été répercutée par le NPD-Québec à quelques reprises après 1985, notamment à l'occasion de la présentation du mémoire du NPD-Québec devant la Commission parlementaire sur l'Accord du Lac Meech, en mai 1987. ${ }^{20}$ Mais cette orientation est demeurée une position du seul NPD-Québec. Elle n'a jamais été partagée par les dirigeants fédéraux et ceux-ci n'en ont jamais tenu compte dans le dossier constitutionnel. À l'occasion du congrès fédéral de 1987, alors que la question constitutionnelle devenait l'enjeu des délibérations, le NPD-Québec a d'autre part demandé que le droit du peuple québécois de déterminer librement son avenir soit reconnu dans la constitution canadienne. Dans la version finale de la résolution adoptée au congrès, négociée sous la pression de la direction fédérale du parti, la référence au droit du peuple québécois de déterminer son avenir a été renvoyée en préambule, dans les attendus de la résolution. ${ }^{21}$ II n'était plus question, selon l'esprit de la résolution finale, de faire reconnaitre ce droit dans la constitution canadienne. En retour, la direction fédérale du parti, supportée par le congrès, admettait reconnaître le caractère «unique» du Québec. Elle acquiesçait aussi à la demande du NPD-Québec voulant que le Québec ait le droit de retrait, avec compensation financière, sur des amendements constitutionnels

19. Constitution québécoise et assemblée constituante, Cabier des résolutions, Congrès du NPD-Québec tenu à Montréal les 7 et 8 septembre 1985, p. 5-7.

20. Mémoire du Nouveau Parti démocratique du Québec présenté par Monsieur Jean-Paul Harney, chef du NPD-Québec, devant la Commission parlementaire sur l'accord du Lac Meech, Québec, le mardi 19 mai 1987.

21. Voir le Cabier des résolutions soumises au congrès du NPD fédéral des 13 , 14 et 15 mars 1987 ; p. 147 pour la résolution du NPD-Québec; p. 143 pour la résolution présentée par le Conseil fédéral (en réplique à celle de l'aile québécoise). 
qui auraient pour effet de transférer des pouvoirs provinciaux au gouvernement fédéral. On s'entendait également pour que soit reconnu au Québec un droit de véto sur toute modification aux institutions fédérales qui pourrait porter atteinte à la langue, à la culture française et aux intérêts nationaux du Québec.

Bref, en mars 1987, la direction fédérale du NPD a fait un premier pas en direction du Québec, mais sans démordre de ses positions fondamentales.

Sur le dossier de la langue, les démêlés entre l'aile québécoise et l'aile fédérale du NPD ont aussi été importants.

En 1986, le Conseil national du NPD-Québec s'est d'abord prononcé en faveur de la défense de la loi 101 et du fait français au Québec. Mais à ce moment-là, il laissait ouverte la possibilité d'usage d'une autre langue que le français dans l'affichage, dans la mesure où le français restait prioritaire. ${ }^{22}$ Cependant, devant la résurgence du débat linguistique, le congrès du NPD-Québec de novembre 1987 a modifié cette position. Il a réaffirmé son appui total à la loi 101 et s'est prononcé en faveur de l'affichage unilingue français. Dans la même veine, le NPD-Québec s'est associé aux diverses campagnes et manifestations publiques en faveur de la loi 101. Les dirigeants fédéraux du parti, Ed Broadbent en particulier, ne se sont pas immiscés dans ce débat, laissant l'aile québécoise déterminer sa propre position sur la loi 101.

Les divergences entre le NPD-Québec et le parti fédéral ont été plus marquantes lorsqu'il s'est agi de trancher la question des prérogatives législatives en matière de langue.

Au congrès fédéral de mars 1987 , dans sa résolution constitutionnelle, le NPD-Québec a demandé que «la constitution canadienne reconnaisse au Québec le droit exclusif de légiférer en

22. Pour la défense de la loi 101 et du franł̧ais au Québec, Déclaration du NPDQuébec, 1986, p. 5. 
matière linguistique, dans le respect de ses minorités». Avant le congrès, cette demande avait déjà suscité l'opposition de plusieurs dirigeants du parti, dont Ed Broadbent et Marion Dewar, exprésidente du NPD. Au moment du congrès, après des tractations en ce sens, la résolution du NPD-Québec a été retirée et remplacée par celle du Conseil fédéral proposant que le parti «explore de nouvelles dispositions constitutionnelles dans le but de protéger les droits linguistiques de la majorité du Québec et d'assurer la préservation des droits linguistiques des minorités tels qu'ils existent présentement dans la constitution».

Ce dernier engagement d'exploration n'a pas fait long feu. Quelques semaines plus tard, à l'opposé de l'orientation du NPDQuébec, les dirigeants fédéraux du NPD appuyaient l'Accord du Lac Meech. Dans le sens de la position soutenue par le NPD fédéral, l'Accord du Lac Meech prévoit une compensation financière (une «juste» compensation) aux provinces qui exerceraient leur droit de retrait sur une modification entraînant un transfert de compétences législatives provinciales au Parlement fédéral. Il permet aussi à chaque province, dont le Québec, de bloquer toute modification aux institutions fédérales. Mais l'appui donné par le NPD à cet accord a rapidement mis fin à son engagement d'explorer une meilleure protection des droits de la majorité française du Québec. L'Accord du Lac Meech ne prévoit, en effet, aucune disposition visant une meilleure protection de la langue française au Québec. Certains observateurs avisés estiment même que cet Accord aura l'effet contraire ${ }^{23}$, compte tenu de la nouvelle règle interprétative qu'il introduit et d'autant plus que l'entente ne fait nulle mention de l'existence du peuple québécois, constitué d'une majorité française. L'Accord du Lac Meech ne reconnaît pas non

23. C'est notamment l'opinion rendue par Michel Plourde dans le récent ouvrage intitulé La politique linguistique du Québec de 1977 à 1987, Montréal, Institut québécois de recherche sur la culture, 1988, p. 110-111. 
plus au Québec, de toute évidence, son droit à l'autodétermination, comme le réclame le NPD-Québec depuis 1985. Enfin, l'Accord permet l'intrusion du pouvoir fédéral dans les domaines de juridiction des provinces par le biais du pouvoir de dépenser. Reflétant ses positions centralisatrices, cette dernière dimension de l'accord du Lac Meech a particulièrement été soutenue par la direction du NPD fédéral.

Compte tenu de ces faits, les désaccords entre l'aile fédérale et l'aile québécoise du parti sur la question nationale se sont cristallisés autour de l'Accord du Lac Meech. L'aile fédérale a donné son feu vert à l'Accord tandis que le Conseil national du NPD-Québec, en mai 1987, a officiellement fait part de son opposition. Par la suite, les divergences se sont étendues à la nouvelle Loi sur les langues officielles. Tous ces désaccords ont nourri des dissentions et des divisions à l'intérieur même du NPDQuébec.

Toutefois, à compter du congrès du NPD-Québec de novembre 1987, les dirigeants du NPD-Québec ont cherché à éviter toute polémique publique à ce sujet. Par exemple, contrairement à ce qui était prévu, le congrès de novembre 1987 ne s'est pas prononcé sur l'Accord du Lac Meech. Par un artifice de procédure, le débat sur cette question a tout simplement été écarté par la direction du NPD-Québec. ${ }^{24}$ Cet incident, survenu au dernier congrès du NPD-Québec, est fort révélateur de l'évolution toute récente de l'aile québécoise. Après s'être d'abord démarquée de l'aile fédérale sur la question nationale, la direction du NPD-Québec a déployé une stratégie de plus en plus conciliante et timorée sur cet enjeu. Plus l'échéance des élections fédérales s'est rapprochée, plus les

24. La présidence du congrès était sous la responsabilité de Michel Agnaieff, responsable fédéral du NPD-Québec et associé à l'aile fédéraliste du NPD-Québec. Au moment prévu du débat, invoquant le manque de temps avant la tenue des élections à la chefferie, celui-ci a ajourné la séance. 
dirigeants du NPD-Québec ont mis la pédale douce sur leurs propres positions sur la question nationale. Plus ils ont multiplié les détours et les compromis de façon à éviter toute confrontation avec l'aile fédérale du parti. Les déclarations d'allégeance envers le fédéralisme ont été aussi plus fréquentes. Cet enlignement stratégique était déjà perceptible au moment du congrès fédéral de mars 1987. Mais il l'était davantage encore au congrès du NPD-Québec de novembre 1987.

Dans la même veine, le débat sur la structure unique du parti a aussi été écarté, du moins jusqu'à ce que le débat ne surgisse à nouveau, comme ce fut le cas au congrès de septembre 1985. Or, la structure unique de l'organisation québécoise peut vraisemblablement agir sur la construction du NPD au Québec. Cette question s'est avérée et reste une question controversée dans les rangs du parti depuis 1985. En effet, lorsqu'a été entreprise la relance du NPD-Québec, ses dirigeants ont opté pour une structure unique du parti au Québec, agissant à la fois aux deux niveaux, provincial et fédéral, structure qui leur a fourni de plus grands moyens matériels. Cette structure unique prévoit, par ailleurs, une double structure à la base du NPD-Québec, avec des associations de comté distinctes au plan fédéral et au plan provincial. L'adhésion au NPD-Québec est donc à la fois une adhésion au NPD fédéral et à l'organisation provinciale. Le congrès de septembre 1985 a retenu cette option. À cette occasion, les partisans d'une double structure du NPD au Québec, l'une fédérale, l'autre provinciale, ont été mis en minorité. Depuis, ils ont pour la plupart quitté le NPD-Québec. La question a été soulevée à nouveau à l'occasion de la démission de Robert Toupin. Mais elle n'a pas été présente dans les débats au congrès de novembre 1987. Cependant, cette structure unique peut s'avérer un obstacle à la progression du NPD au Québec. Elle peut évidemment rebuter ceux qui voudraient adhérer au parti fédéral sans adhérer au parti provincial, comme ce fut le cas pour un certain nombre de sup- 
porteurs du Parti québécois. Mais plus fondamentalement, la structure unique du NPD-Québec pourrait s'avérer un obstacle à son développement comme parti œuvrant sur la scène proprement québécoise. Plus particulièrement depuis les années 1960, la réalité de la question nationale québécoise a peu à peu obligé tous les grands partis évoluant au Québec à se doter d'une structure distincte des partis fédéraux au risque de se couper de tout support populaire. Les vieux partis ont déjà dû, bon gré mal gré, s'adapter à cette spécificité du Québec. Un système partisan propre au niveau québécois s'est donc peu à peu développé, à cause de la question nationale. Le NPD-Québec, croyons-nous, pourra difficilement échapper à cette problématique, d'autant plus que des divergences importantes subsistent avec l'aile fédérale du NPD. Dans l'avenir, cette question rebondira probablement dans les délibérations du NPD-Québec.

En résumé, la question nationale n'est aucunement réglée dans les rangs du NPD. Les désaccords, même étouffés, persistent. Ils risquent, comme par le passé, d'influencer l'évolution future du parti. Mais au-delà des divergences et malgré la mince ouverture manifestée par le NPD fédéral face aux aspirations nationales du peuple québécois, les dirigeants de l'aile québécoise ont quand même cherché, après 1985, à profiter de la vague de popularité du NPD.

Évitant bientôt toute prise de position qui aurait eu pour effet de gêner l'aile fédérale, usant d'une certaine dose d'opportunisme, mettant toutes les énergies dans le travail d'organisation, les dirigeants du NPD-Québec ont ainsi escompté se placer en position favorable pour les élections fédérales anticipées. Les progrès réalisés par le NPD au Québec sont l'objet de la dernière partie de notre article. 


\section{La progression du NPD au Québec}

Après 1984 , le NPD a bel et bien réalisé des progrès indéniables au Québec. En effet, en mars 1984, avant la venue de Jean-Paul Harney à la direction du NPD-Québec, l'organisation néo-démocrate québécoise ne comptait plus que quelques dizaines de membres. Elle était sans exécutif. Tout était à refaire, à reconstruire. Depuis ce temps, un travail de relance a été impulsé, marqué par un effort de restructuration et de recrutement. Au cours de l'année 1985, deux congrès du NPD-Québec ont servi de point d'appui à l'amorce de la réorganisation du parti. Un troisième congrès a été tenu en novembre 1987. Les pas franchis méritent une analyse particulière.

Au plan organisationnel, le NPD-Québec a certainement réalisé les progrès les plus significatifs. Le NPD-Québec a surtout connu une progression quant à son membership. Ce développement est à signaler, si on le compare à l'héritage néo-démocrate au Québec. Avec la campagne électorale fédérale de 1984, le NPDQuébec a d'abord réussi à regrouper un premier bassin d'un millier de membres environ. Par la suite, la progression a été plus marquée, surtout à compter de 1987. D'octobre 1984 à mars 1987, selon les données publiques fournies par le NPD-Québec, le membership de l'organisation néo-démocrate est passé de 1,400 à 3,225. En novembre 1987 , il passait à 7,000 . En mars 1988 , ce nombre grimpait à 12,000 . À la veille de la campagne électorale fédérale, le membership frisait les 16,000 adhérents, un nombre que le NPD-Québec n'a jamais atteint après sa fondation en 1961.

Peu à peu, le NPD-Québec s'est assuré d'une implantation dans plusieurs régions du Québec: dans la région de Montréal, celle du Québec métropolitain, dans l'Outaouais, dans la Mauricie, dans la région du Lac Saint-Jean. Les associations fédérales locales du NPD-Québec ont été multipliées, avec l'objectif d'assurer la présence du NPD-Québec dans chacune des 75 circonscriptions 
de la province. S'appuyant sur sa progression numérique, le NPDQuébec s'est développé un nouveau noyau de dirigeants et de militants. Parmi eux, on retrouve un bon nombre de syndicalistes et d'ex-membres du Parti québécois. ${ }^{25}$ Le NPD-Québec, par ailleurs, a développé ses moyens organisationnels et techniques, dont l'engagement de quelques permanents et l'occupation de nouveaux locaux au siège central de l'organisation à Montréal. Dans une large mesure, cette consolidation des moyens mis à la disposition du NPD-Québec a été rendue possible grâce à l'aide financière du parti fédéral. À la veille de l'élection fédérale de 1988, le NPD entendait même fournir 25 organisateurs au NPD-Québec et dépenser quelques deux millions de dollars au Québec, au cours de la campagne électorale.

D'autre part, le NPD-Québec s'est affairé à rechercher des candidats de prestige pour les élections fédérales. Cette opération n'a pas rapporté tous les résultats escomptés, mais quelques personnalités ont joint l'organisation et ont accepté de se porter candidat aux élections fédérales. Pensons, par exemple, à Philip Edmunston dans Chambly, à Pierre Hétu dans Châteauguay, à François Beaulne dans Laurier-Sainte-Marie, ou encore à Éric Gourdeau dans Montmorency-Orléans, lui-même ex-conseiller du premier ministre René Lévesque. Toutefois, reflétant les dissentions internes au sein de l'organisation néo-démocrate, les déclarations faites par Éric Gourdeau à la veille de la campagne électorale fédérale (associant les dirigeants du NPD-Québec à un groupe de philosophes trotskistes et léninistes) n'ont certainement pas aidé la cause publique du NPD-Québec, tout comme les accusations semblables lancées quelques mois auparavant par l'ex-député conservateur Robert Toupin.

25. Comme l'a révélé une enquête effectuée par le NPD-Québec auprès des délégués présents au congrès des 27, 28 et 29 novembre 1987. 
Toujours au plan fédéral, le NPD-Québec a également reçu l'appui de la FTQ. Cet appui, survenu au moment même du déclenchement de la campagne électorale fédérale, est important. La FTQ s'engageait même à libérer une trentaine de permanents syndicaux durant la campagne électorale. Après 1965, jamais la FTQ n'a manifesté un appui comparable au NPD à l'occasion d'une élection fédérale.

Comme on le constate, c'est donc surtout en fonction de la scène politique fédérale que le NPD-Québec a connu une progression et a vu ses appuis se renforcer. Il en est tout autrement sur la scène proprement québécoise.

Outre le problème posé par la question nationale et la structure unique du parti, la faiblesse du NPD-Québec sur la scène politique provinciale s'est manifestée notamment au plan du leadership.

En 1987, après que Jean-Paul Harney eût annoncé qu'il ne se représentait pas à la chefferie du parti, le NPD-Québec a dû choisir une nouvelle direction et un nouveau chef. Dans cette foulée, Roland Morin a été élu chef du NPD-Québec au congrès de novembre 1987. Peu connu du grand public, Roland Morin est un vieux routier du parti, membre du NPD depuis 1963. Candidat et battu à six reprises dans les élections fédérales et provinciales, il a été notamment leader du NPD-Québec en 1970 et président du NPD-Québec de 1985 à 1987. En bout de ligne, la candidature et l'élection de Roland Morin comme chef du parti n'ont pas suscité d'enthousiasme dans les rangs du parti, même si l'aile nationaliste du NPD-Québec supportait sa candidature. Cet événement n'a pas généré non plus de grands changements quant à l'image et l'impact du parti parmi la population, d'autant plus que la présence publique du NPD-Québec, sous l'égide de son nouveau leadership, s'est peu manifestée. L'élection provinciale partielle du 20 juin 1988 dans le comté d'Anjou en a été la démonstration la plus évidente. Roland Morin n'a recueilli que 483 voix, soit $2,24 \%$ des voix exprimées. L'absence d'un leader 
de renommée, capable de susciter l'appui de la population, reste une carence majeure du NPD-Québec.

Mais ce qui vient renforcer doublement la faiblesse du NPDQuébec sur le terrain proprement québécois, c'est qu'il ne peut compter sur l'appui direct du mouvement syndical, si ce n'est quelques organisations syndicales locales et de nombreux syndicalistes, à titre individuel. La direction de la FTQ reste opposée à la présence du NPD-Québec sur la scène politique québécoise ${ }^{25}$. La CSN, pour sa part, s'en tient à sa position traditionnelle, dite non-partisane. Quant à la CEQ, sa position va dans le même sens que la CSN, bien que quelques-uns de ses dirigeants ou permanents soient activement impliqués dans le NPD-Québec. Conséquemment, sur le plan de ses rapports avec le mouvement syndical, le NPD-Québec se trouve dans une position tout à fait différente de celles des autres sections du NPD, qu'il s'agisse de l'aile fédérale ou des autres partis provinciaux néo-démocrates, en Ontario ou dans l'Ouest canadien. Cette carence, à notre avis, sans être une déterminante absolue, affaiblit grandement le travail de relance du NPD-Québec. Car au Canada anglais ou en Europe, en Suède, en Norvège ou en Grande-Bretagne, les grands partis sociauxdémocrates ont la force qu'on leur connaît notamment grâce au support du mouvement syndical.

Conséquemment, la performance du NPD sur la scène électorale québécoise a été à la mesure de ses faiblesses. Aux élections du 2 décembre 1985, les 90 candidats du NPD-Québec ont recueilli quelque $2,48 \%$ du suffrage total exprimé $(3,25 \%$ sur la base des 90 comtés où le NPD-Québec présentait des candidars).

25. Au congrès de la FTQ de décembre 1987, les dirigeants de la centrale ont clairement fait savoir leur position. Ils l'ont reconfirmé lorsqu'ils ont annoncé leur appui au NPD à l'occasion de l'élection fédérale. Les liens passés entre les dirigeants de la FTQ et le Parti québécois sont bien connus. Il semble que la présence du Parti québécois y soit encore pour quelque chose. 
En janvier 1986, à l'élection partielle de Saint-Laurent, le candidat néo-démocrate a réussi à améliorer légèrement la performance du NPD, recueillant $9,7 \%$ des voix et se classant deuxième après Robert Bourassa. Par la suite, en 1987, certains sondages ont attribué entre 15 et $20 \%$ des voix au NPD-Québec advenant une élection provinciale. Toutefois, le renforcement du Parti québécois, avec Jacques Parizeau à la tête du parti, s'est fait au détriment du NPD-Québec. Au début de l'année 1988, les intentions de vote en faveur du NPD-Québec auraient chuté à $12 \%$ selon la maison Créatec, et à $9 \%$ selon la maison Crop. ${ }^{26}$ Ainsi, peu à peu, sur la scène électorale québécoise, la progression du NPD-Québec s'est révélée fragile et très incertaine. Puis, le 20 juin 1988, dans Anjou, le NPD-Québec a littéralement échoué. ${ }^{27}$ Il a fait un retour au score de 1985 .

\section{Conclusion}

De 1984 à 1988, le NPD a définitivement renforcé ses positions sur la scène politique fédérale, profitant de circonstances tout à fait particulières. Le NPD s'est appuyé sur un fort mouvement d'insatisfaction à l'égard des deux vieux partis parmi une frange importante de la population canadienne et québécoise. Cette réprobation nous est apparue plus forte que ce qu'elle était de 1958 à 1961, au moment de la fondation du NPD. Le NPD a tenté de capitaliser et de gagner l'électorat, notamment au Québec. Pour y parvenir, le parti fédéral a fait preuve d'une petite ouverture sur la question nationale québécoise, bien que ses positions n'aient pas radicalement changé.

26. Nous faisons référence ici au sondage Créatec tenu du 13 au 16 janvier 1988 et au sondage Crop tenu du 15 au 20 janvier.

27. Pour l'élection partielle de Roberval, le NPD-Québec ne présentait aucun candidat. 
Au plan fédéral, le phénomène d'érosion du bipartisme, amorcé en 1961, est donc devenu une réalité évidente. La lutte électorale fédérale de 1988 est devenue une bataille à trois, comme les élections l'ont été en Ontario au cours des dernières années. Dès le début de la campagne électorale de 1988, il apparaissait que le NPD était en mesure de dépasser la barre du $20 \%$ des voix et la trentaine de députés. Il était déjà à prévoir que le NPD renforce ses positions, y compris au Québec. À l'ouverture de la campagne électorale de 1988, il n'était pas interdit de penser que pour une première fois dans son histoire, le NPD pourrait faire élire des candidats au Québec, bien que cette probabilité reste plutôt faible.

La croissance des appuis électoraux du NPD fédéral en territoire québécois ne garantit aucunement, par ailleurs, la construction du NPD-Québec comme parti de masse sur la scène politique québécoise. Au Québec, d'autres considérations interviennent. Elles renvoient à la question nationale, à la structure unique du parti, à la présence du Parti québécois, au rôle du mouvement syndical et à l'absence d'un leader de renommée susceptible de gagner un appui populaire. Sur ce terrain, les obstacles que rencontre le NPD-Québec sont énormes. Sa performance, de 1984 à 1988, en est un reflet on ne peut plus évident. 